\title{
Evaluation of Carbamazepine Pharmacotherapy In Patients With Trigeminal Neuralgia
}

\author{
Syed Othman SNI ${ }^{1}$, Mazlam NA ${ }^{1}$, Kallarakkal TG ${ }^{2}$ \\ ${ }^{1}$ Faculty of Dentistry, University of Malaya, Kuala Lumpur, Malaysia. \\ ${ }^{2}$ Department of Oral and Maxillofacial Clinical Sciences, Faculty of Dentistry, University of Malaya, \\ Kuala Lumpur, Malaysia.
}

\begin{abstract}
In this study we aimed to determine the adverse effects and pain outcome following carbamazepine pharmacotherapy to manage patients with classical trigeminal neuralgia. The study was carried out on patients diagnosed with classical trigeminal neuralgia at the Faculty of Dentistry, University of Malaya. Medical records of patients diagnosed with trigeminal neuralgia over a period of 11 years from April, 2003 to April, 2014 were reviewed. A total of 49 patients who fulfilled the inclusion criteria were included in the study. Demographic details, clinical features and blood test reports of the patients were recorded from the patient's clinical files. Subjects included in the study were administered the Faces Pain Rating Scale (FPS) and Numeric Pain Rating scale (NRS) to measure the pain outcome retrospectively. Analysis and interpretation of results was carried out using SPSS. Hepatic adverse effects were more commonly seen in our patients $(n=39)$ followed by haematological $(n=27)$, renal $(n=23)$ and non-specific adverse effects $(n=22)$. However, there was no significant association between the observed side effects and carbamazepine pharmacotherapy. Analysis of our data using Pearson Chi Square test, showed no significant correlation between gender, ethnicity and age of the patients with adverse effects. 9 out of the 49 subjects included in the study were administered the FOS and the NRS. Pain perception after treatment was significantly decreased ( $p-0.007)$ in comparison to pain perception before commencement of therapy with carbamazepine in the selected group of patients. Even though pain perception decreased in the selected patients, the data is insufficient to state the efficacy of the treatment as none of the patients reported a complete relief from their pain. The results of our study showed that there were no significant haematological, hepatic, renal, and non-specific adverse effects associated with the use of carbamazepine. The efficacy of this drug in the treatment of trigeminal neuralgia was proven to be satisfactory. It may be concluded that carbamazepine could be considered as the drug of choice in the treatment of trigeminal neuralgia.
\end{abstract}

Keywords: Classical trigeminal neuralgia, carbamazepine, hepatic adverse effects, renal adverse effects

\section{Introduction}

Trigeminal neuralgia also known as Tic Douloureaux is a chronic pain condition that affects the trigeminal or $5^{\text {th }}$ cranial nerve. Trigeminal neuralgia is a sudden, severe nerve pain that is usually described as a stabbing, piercing or an electric shock- like pain. The aetiology of trigeminal neuralgia is not clearly known. In most cases, it is due to compression of the trigeminal nerve root close to its point of entry into the pons by an aberrant loop of artery or vein 
(1). According to the International Headache Society (HIS), trigeminal neuralgia can be classified into idiopathic and symptomatic trigeminal neuralgia (2). In idiopathic trigeminal neuralgia no cause of symptoms can be identified other than vascular compression. Symptomatic trigeminal neuralgia has the same clinical criteria but another underlying cause is responsible for the symptoms (3).

The incidence of trigeminal neuralgia is rare, affecting mostly females and predominantly seen in people between 60 to 70 years of age. It usually affects only one side of the face (unilateral) but it can also occur bilaterally. Trigeminal neuralgia occurs spontaneously or is triggered by non - noxious stimuli applied to a specific trigger point. The trigger point is usually located in the nasolabial fold, upper lip, lower lip and gingiva but it also can occur anywhere within the area of the distribution of the trigeminal nerve (2).

The initial treatment of choice for trigeminal neuralgia is medical therapy. Patients who do not respond or who relapse with medical therapy should be considered for surgical treatment. Surgery may also be considered for patients who are intolerant to medical treatment (3). Pharmacologic treatments for trigeminal neuralgia are limited. Most of the drugs used in the treatment of trigeminal neuralgia interfere with ion channels and block the pain that is caused by ectopic neuronal firing. Additionally, some of the drugs may inhibit noxious transmission by reducing glutamate release and by augmenting GABAergic inhibition (4). Carbamezapine is the first line of treatment in patients with trigeminal neuralgia. Alternative pharmacologic treatment modalities include lamotrigene, baclofen, phenytoin, clonazepam, valproic acid and gabapentin (5). Patients on treatment with carbamezapine show a broad spectrum of side effects such as dizziness, ataxia, drowsiness and reduction of alertness particularly in the beginning of treatment and with increasing doses which improve some days after the drug has reached a stable level. Carbamazepine induced skin rash and serious adverse reactions such as haematologic toxicity occur rarely. Haematologic toxicity usually manifests as neutropenia, thrombocytopenia, agranulocytosis and aplastic anaemia particularly in high risk patients. Similarly, other pharmacologic interventions have also been reported to cause adverse effects (6).

Surgical procedures to treat trigeminal neuralgia may be percutaneous or open. The choice of procedure should be made after the potential risks and benefits of each procedure have been evaluated. Percutaneous techniques include glycerol injection, balloon compression, radiofrequency rhizotomy, and gamma knife stereotactic radiosurgery. These techniques are relatively noninvasive outpatient procedures or require only a short hospital stay, and lack life threatening adverse effects. Open techniques include partial trigeminal rhizotomy and microvascular decompression. These procedures involve posterior fossa exploration with its attendant risks, including stroke, meningitis, and death (3).

Pain is a complex and subjective experience that is difficult to measure primarily due to its multifaceted and subjective nature. Proper outcome measures are needed to scientifically validate treatment efficacy. There is no valid and reliable method to objectively quantify an individual's experience of pain. Clinicians mostly rely on selfreported measures to determine the impact of pain. Unidimensional pain scales used to measure pain outcome are simple, quick to administer and easily understood by the patient. These include the numerical rating scale (NRS), visual analogue scale (VAS) and the verbal rating scale (VRS). A commonly used unidimensional tool is the numerical rating scale (NRS). Although variations exist, the instrument typically consists of scores from 0 to 10 (or 0-100), with the far left being described as "no pain" and the far right described as "worst pain imaginable. The NRS has the advantage of being administered verbally, thus not requiring patient mobility. The VAS is considered to be the most sensitive single-item measure for clinical pain research whereas the VRS has several statistical drawbacks and is used only when patient characteristics require it $(7,8)$

Neuropathic pain is a pain arising as a direct consequence of a lesion or disease affecting the somatosensory system. Several tools essentially based on pain descriptors have been proposed for the purpose of distinguishing neuropathic pain from non-neuropathic pain. These tools include McGill Pain Questionnaire (MPQ), The Leeds Assessment of Neuropathic Symptoms and Signs (LANSS), The Neuropathic Pain Questionnaire (NPQ), The Douleur Neuropathique en 4 questions (DN4), PainDETECT, ID Pain and The standardized evaluation of pain (StEP) (9). The faces pain scale (FPS) has been shown to be a valid and sensitive scale for assessing pain intensity in the general population. The use of FPS to measure pain intensity avoids language and many cross- cultural differences. It consists of a series of cartoon like faces that represent varying degrees of pain. The FPS was originally developed for use in paediatrics but it has also been found to be valid and reliable for use in adults. It has been found to be effective in cognitively-impaired and end-of-life non-verbal individuals as it does not require reading, writing or energy for extensive expression. There are several versions of the FPS that come with six, seven or nine faces (10). 
The therapeutic effect of carbamazepine is prompt and most patients experience pain relief in 48 hours but the effect may diminish over time. Even though monotherapy is preferable, polypharmacy with other anti-epileptic medications may be required for the patient to remain pain free. Side-effects that may arise with the use of carbamazepine may be minimized by carefully titrating the doses throughout the day. Therefore, in this study we aimed to determine the adverse effects of pharmacotherapy with carbamazepine employed to manage patients with classical trigeminal neuralgia. In addition, we also aimed to determine the pain outcome in patients undergoing pharmacological treatment with carbamazepine for classical trigeminal neuralgia as a measure to evaluate the efficacy of treatment. The findings from this study may be used to recommend modifications in the management of patients with classical trigeminal neuralgia by pharmacotherapy.

\section{Materials and Methods}

The study was retrospectively carried out on patients diagnosed with classical trigeminal neuralgia in the Oral Medicine clinic of the department of Oral and Maxillofacial Clinical Sciences at the Faculty of Dentistry, University of Malaya. Medical records of patients diagnosed with classical trigeminal neuralgia over a period of 11 years from April, 2003 to April, 2014 and who were under regular followup in the Oral Medicine clinic were reviewed. The study protocol was approved by the Medical Ethics Committee at the Faculty of Dentistry, University of Malaya [Ethics DF OS1416/0044(U)].The following were the inclusion and exclusion criteria for selection of patients into the study;

\section{Inclusion criteria:}

1. Patients diagnosed with trigeminal neuralgia and who fulfilled the International classification of Headache Disorders II (ICHD-II) criteria for classical trigeminal neuralgia

2. Patients with complete medical records

\section{Exclusion criteria:}

1. Patients with a history of mental illnesses, blood dyscrasias, liver and kidney disease.

2. Patients who were on any other systemic medications that may have produced CNS suppression, haematologic toxicity, fluid retention and impaired liver function
Demographic details and clinical features of the patient's condition were recorded from the patient's clinical files. Specific information that denoted features suggestive of CNS suppression and adverse skin reactions observed at initiation of pharmacotherapy were recorded. The patient's blood test reports within the first six months of initiation of pharmacotherapy as recorded in the patient's clinical files were evaluated to determine features suggestive of haematologic toxicity, impaired liver and renal function besides other nonspecific adverse effects. Subjects included in the study were administered the faces pain scale (FPS) and (NRS) (Figure 1) to measure the pain outcome retrospectively as perceived by the patient prior to the initiation of therapy and prospectively at the last documented follow-up. Analysis and interpretation of results were carried out using SPSS 16.00 statistical software.

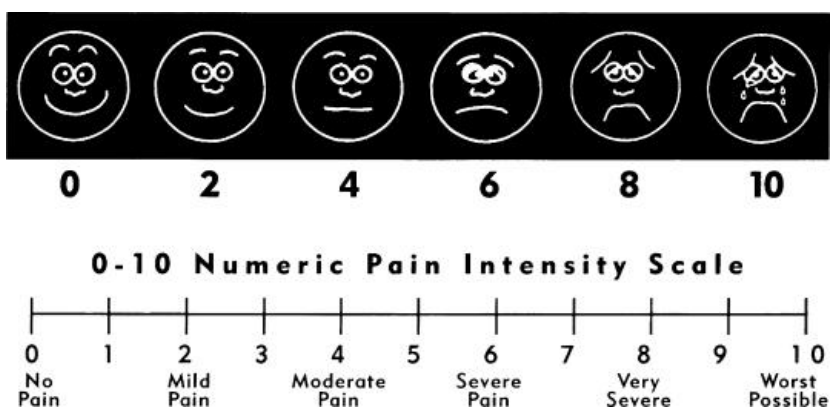

Figure 1: Wong/Baker faces rating scale and the numeric pain intensity scale (11)

\section{Results}

Clinical files of 49 patients with complete medical records that included blood test reports, renal function test and liver function test reports ascertained during the first 6 months of initiation of pharmacotherapy were included in our study. Our results showed that majority of the patients in our study were females (65.3\%). Chinese patients $(46.9 \%)$ constituted the majority of our cases followed by Malays (40.8\%). The median age of our patients was 64 years. The right side of the face was found to be predominantly affected in majority of our cases (63.3\%). Involvement of the maxillary and mandibular divisions of the trigeminal nerve showed an equal distribution $(36.7 \%)$ in our cases.

\section{Distribution of adverse effects among cases:}

Hepatic adverse effects were more commonly seen in our patients $(n=39)$ followed by haematological $(n=27)$, renal $(n=23)$ and non-specific adverse 
effects ( $n=22)$ (Figure 2). Renal adverse effects were observed to occur more commonly in females but it was not found to be statistically significant $(p=0.073)$. The median age of the patients in our study was 64 years old. Renal adverse effects were found to more common in patients aged $\leq 64$ years although it was not statistically significant $(p=0.069)$. Analysis of our data using Pearson Chi Square test, showed no significant correlation between gender, ethnicity and age of the patients with haematological, hepatic, renal and non-specific adverse effects.

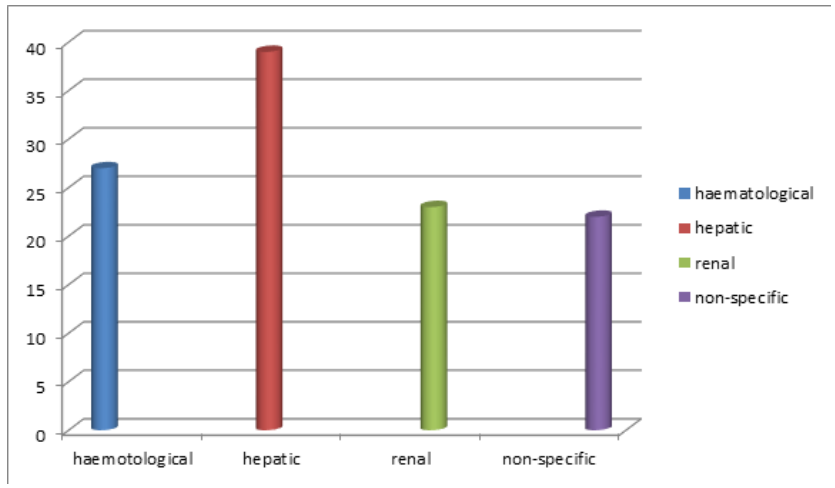

Figure 2: Bar graph showing the distribution of various adverse effects among the cases

\section{Correlation of adverse effects with dosage of carbamazepine therapy}

The daily dosage of carbamazepine in our patients ranged from $100-600 \mathrm{mg}$ with majority of the patients being administered $200 \mathrm{mg}$ daily. Hence we categorised our patients into two groups;

Low dosage group- comprising of patients on a maximum daily dosage of up to $200 \mathrm{mg}$ of carbamazepine.

High dosage group- comprising of patients on a daily dosage of carbamazepine ranging between $200 \mathrm{mg}$ to a maximum dosage of $600 \mathrm{mg}$.

\section{Correlation of adverse effects with dosage}

There was a significant correlation between relative distribution width (RDW) and daily dosage in patients included in the high dosage group $(p=0.029)$ (Table 1).

Results of Pearson Chi Square test showed a significant correlation $(p=0.027)$ between elevated levels of serum globulin and daily dosage of carbamazepine in patients within the low dosage group (Table 2). There was no significant correlation between renal adverse effects and daily dosage of carbamazepine.
Table 1: Correlation of haematological adverse effects with dosage

\begin{tabular}{|c|c|c|c|c|}
\hline \multirow{2}{*}{$\begin{array}{l}\text { Haematological } \\
\text { Adverse Effect } \\
\text { Low }\end{array}$} & & \multicolumn{2}{|c|}{$\begin{array}{c}\text { Dosage of drugs } \\
\text { used }\end{array}$} & \multirow[t]{2}{*}{ P Value } \\
\hline & & High & & \\
\hline \multirow[t]{2}{*}{ Eosinophils \% } & Yes & 100.0 & 0.00 & 0.086 \\
\hline & No & 46.8 & 53.2 & \\
\hline \multirow[t]{2}{*}{ Haemoglobin } & Yes & 42.9 & 57.1 & 0.726 \\
\hline & No & 50.0 & 50.0 & \\
\hline \multirow[t]{2}{*}{ Haematocrit } & Yes & 41.7 & 58.3 & 0.560 \\
\hline & No & 51.4 & 48.6 & \\
\hline \multirow[t]{2}{*}{ RDW } & Yes & 23.1 & 76.9 & 0.029 \\
\hline & No & 58.3 & 41.7 & \\
\hline \multirow[t]{2}{*}{ RBC } & Yes & 50.0 & 50.0 & 0.957 \\
\hline & No & 48.8 & 51.2 & \\
\hline \multirow[t]{2}{*}{$\mathrm{MCH}$} & Yes & 50.0 & 50.0 & 0.942 \\
\hline & No & 48.7 & 51.3 & \\
\hline \multirow[t]{2}{*}{ WBC } & Yes & 66.7 & 33.3 & 0.524 \\
\hline & No & 47.8 & 52.2 & \\
\hline \multirow[t]{2}{*}{ Lymphocyte } & Yes & 0.00 & 100.0 & 0.243 \\
\hline & No & 50.0 & 50.0 & \\
\hline \multirow[t]{2}{*}{$\mathrm{MCHC}$} & Yes & 50.0 & 50.0 & 0.966 \\
\hline & No & 48.9 & 51.1 & \\
\hline \multirow[t]{2}{*}{ Platelet count } & Yes & 37.5 & 62.5 & 0.475 \\
\hline & No & 51.2 & 48.8 & \\
\hline \multirow[t]{2}{*}{ Basophils } & Yes & 100.0 & 0.00 & 0.086 \\
\hline & No & 46.8 & 53.2 & \\
\hline \multirow[t]{2}{*}{ MCV } & Yes & 50.0 & 50.0 & 0.957 \\
\hline & No & 48.8 & 51.2 & \\
\hline
\end{tabular}

Table 2: Correlation of hepatic adverse effects with dosage

\begin{tabular}{lllll}
\hline \multirow{2}{*}{$\begin{array}{l}\text { Hepatic Adverse Effect } \\
\text { Low }\end{array}$} & \multicolumn{2}{c}{$\begin{array}{l}\text { Dosage of drugs } \\
\text { used }\end{array}$} & P Value \\
\cline { 3 - 4 } GGT & Yes & 56.3 & 43.8 & 0 \\
\cline { 2 - 3 } Globulin & No & 45.5 & 54.5 & \\
& Yes & 70.6 & 29.4 & 0.027 \\
Albumin & No & 37.5 & 62.5 & \\
& Yes & 100.0 & 0.00 & 0.086 \\
ALP & No & 46.8 & 53.2 & \\
& Yes & 66.7 & 33.3 & 0.351 \\
ALT & No & 46.5 & 53.5 & \\
& Yes & 35.7 & 64.3 & 0.240 \\
AST & No & 54.3 & 45.7 & \\
& Yes & 37.5 & 62.5 & 0.478 \\
Conjugated & No & 51.2 & 48.8 & \\
Billirubin & Yes & 50.0 & 50.0 & 0.976 \\
\hline
\end{tabular}


A significant correlation $(p=0.040)$ was observed in patients on a high dose of carbamazepine and gastrointestinal problems specifically nausea and vomiting (Table 3).

Table 3: Correlation of non-specific adverse effects with dosage

\begin{tabular}{|c|c|c|c|c|}
\hline \multirow{2}{*}{\multicolumn{2}{|c|}{$\begin{array}{l}\text { Non Specific Adverse } \\
\text { Effect } \\
\text { Low }\end{array}$}} & \multirow{2}{*}{\multicolumn{2}{|c|}{$\begin{array}{l}\begin{array}{c}\text { Dosage of drugs } \\
\text { used }\end{array} \\
\text { High }\end{array}$}} & \multirow[t]{2}{*}{ P Value } \\
\hline & & & & \\
\hline \multirow[t]{2}{*}{ Drowsiness } & Yes & 41.7 & 58.3 & 0.560 \\
\hline & No & 51.4 & 48.6 & \\
\hline \multirow{2}{*}{$\begin{array}{l}\text { Skin } \\
\text { itchiness }\end{array}$} & Yes & 50.0 & 50.0 & 0.966 \\
\hline & No & 48.9 & 51.1 & \\
\hline \multirow[t]{2}{*}{ Lethargy } & Yes & 50.0 & 50.0 & 0.966 \\
\hline & No & 48.9 & 51.1 & \\
\hline \multirow[t]{2}{*}{ Skin rashes } & Yes & 50.0 & 50.0 & 0.966 \\
\hline & No & 48.9 & 51.1 & \\
\hline \multirow[t]{2}{*}{ Hair fall } & Yes & 100.0 & 0.00 & 0.229 \\
\hline & No & 47.9 & 52.1 & \\
\hline \multirow{2}{*}{$\begin{array}{l}\text { GIT (nausea } \\
\text { \& vomiting) }\end{array}$} & Yes & 0.00 & 100.0 & 0.040 \\
\hline & No & 52.2 & 47.8 & \\
\hline \multirow[t]{2}{*}{ Dizziness } & Yes & 33.3 & 66.7 & 0.409 \\
\hline & No & 51.2 & 48.8 & \\
\hline \multirow[t]{2}{*}{ Tremor } & Yes & 0.00 & 100.0 & 0.243 \\
\hline & No & 50.0 & 50.0 & \\
\hline \multirow[t]{2}{*}{ Numbness } & Yes & 0.00 & 100.0 & 0.243 \\
\hline & No & 50.0 & 50.0 & \\
\hline \multirow{2}{*}{$\begin{array}{l}\text { Reduced } \\
\text { Appetite }\end{array}$} & Yes & 100.0 & 0.00 & 0.229 \\
\hline & No & 47.9 & 52.1 & \\
\hline
\end{tabular}

\section{Pain Perception}

9 out of 49 patients responded to the pain perception survey. Subjects included in the study were administered the FPS and NRS. The results showed that the pain perception of the patients after the treatment had reduced markedly in comparison to pain perception before the commencement of the pharmacotherapy using the Wilcoxon Signed Ranks Test $(p=0.007)$.

\section{Discussion}

The incidence of trigeminal neuralgia has been reported to be 1 in every 25,000 people (5). Consistent with previous reports in the literature, majority of our patients were elderly females with a median age of 64 years (12). The peak incidence was in the seventh and eighth decades of life in our study. It was observed that trigeminal neuralgia tend to be almost equally distributed between the Chinese and Malays while Indians and others were the least affected groups in our study. These observations were in contrast to the findings from a previous study by Loh et al., which reported an increased incidence of trigeminal neuralgia in the Chinese population. However it has been accounted that the reported increased incidence in the Chinese poulation may be a reflection of the biased racial presentation reported in that series (13). The maxillary and mandibular divisions of the trigeminal nerve were afflicted in the majority of our patients. These findings together with involvement of the right side of the face were consistent with results from previous studies on the Asian and Caucasian populations (13) .

Carbamezapine, an antiepileptic drug is the treatment of choice for trigeminal neuralgia. A major challenge in pharmocotherapy with carbamezapine is side effects that may affect multiple systems. A significant proportion of patients may also eventually becoming refractory to treatment with carbamezapine $(14,15)$.

Haematologic adverse effects are relatively rare but may be potentially life threatening. The haematologic toxicity of carbamezapine is related to epoxides that are released when carbamezapine is metabolized in the liver. The adverse effects include neutropenia, agranulocytosis, eosinophilia, thrombocytopenia, purpura, leukocytosis, thrombocytosis, altered platelet function and anaemia. The incidence of severe haematological adverse effects reported in various studies range from 1:38,000 to 1: 10,800 (16). Haematologic adverse effects usually manifest at the start of the treatment and may last up to about three months. Leukopenia is the most common haematological adverse effect associated with carbamezapine (16). The most severe adverse effect reported is agranulocytosis which may be aggravated in patients with a history of bone marrow depression. Hence it is always recommended to have a baseline CBC before starting treatment. Generally carbamezapine produces a transient reduction in WBCs in atleast $10 \%$ of the patients during the first 4 months of 
treatment. In very rare cases carbamezapine has known to induce pancytopenia (17). It has been widely suggested that carbamezapine induced haematological adverse effects are related to changes in folate metabolism. This may be the result of its ability to induce microsomal liver enzymes or interfere with overall folate absorption (16). As observed in our data $55.1 \%$ of our cases manifested haematological abnormalities. None of our patients developed any blood dyscraias. Majority of our patients with haematological abnormalities demonstrated a tendency towards anaemia as evidenced by decreased haemoglobin concentration, haematocrit, red blood cell count, mean corpuscular haemoglobin, mean corpuscular volume and increased red cell distribution width. A significant correlation was observed between red cell distribution width and patients on a high dosage of carbamezapine $(p=0.029)$. Red cell distribution width is a quantitative marker of variability in the size of erythrocytes. It is a routine assay of complete blood count and is especially a marker in iron deficiency anaemia (18). Similarly $6.1 \%$ of our patients with haematological abnormalities demonstrated decreased WBS counts.

Metabolism of many of the antiepileptic drugs takes place primarily in the liver. Hence liver is subjected to drug induced toxicity which is manifested as a wide range of hepatotoxic reactions ranging from mild tranisent elevations of hepatic enzymes to hepatic failure. Elevation of liver enzymes such as aspartate aminotranferase (AST), alanine aminotranferase, alkaline phosphatase (ALP) and gamma-glutamyl tranferase (GGT) can serve as markers of hepatocellular injury. The hepatotoxicity induced by antiepileptic drugs is usually the result of production of reactive toxic metabolites or induction of immunoallergic reactions and is observed as mild elevations of liver enzymes. These elevations are transient and do not appear to be related to hepatocellular damage. Analysis of our data revealed that $79.6 \%$ of our cases showed hepatic adverse effects as evidenced by asymptomatic alterations of liver function test. $34.7 \%$ of our patients showed increased serum globulin levels while $32.7 \%$ of the patients manifested increased GGT. Increased levels of ALP $(12.2 \%)$, AST (10.87\%), conjugated bilirubin $(4.1 \%)$ and decreased levels of albumin $(4.1 \%)$ were observed in a lesser number of cases. A statistically significant correlation was observed between increased serum globulin levels and patients on low dosage of carbamezapine therapy. Globulins are primarily synthesized in the liver and include carrier proteins, enzymes, complement and immunoglobulins. A polyclonal increase in serum globulin levels may indicate liver disease (19).
None of our patients manifested a more than two to three fold increase in the liver enzymes indicative of hepatocellular toxicity.

Renal complications from carbamezapine are rare and are usually mild and transient. Affected patients predominantly manifest an increase in blood urea nitrogen (BUN), proteinuria, haematuria or glycosuria. Cases have been reported in the literature of more serious carbamezapine induced side effects such as membranous glomerulopathy and acute tubular necrosis (20). Carbamezapine is known to have antidiuretic effects and has been used to decrease urinary volume in patients with diabetes insipidus. Similarly the incidence of hyponatraemia is a common occurence in patients on carbamezapine therapy (21). $46.9 \%$ of our patients exhibited renal adverse effects as manifested by mild alterations in renal function test. None of our patients had evidence of any serious renal damage.

Various non-specific adverse effects have been reprted in the literarture associated with the use of carbamezapine (22). $44.9 \%$ of our patients manifested non-specific adverse effects. A statistically significant correlation was observed between patients on treatment with a high dosage of carbamezapine and gastrointestinal adverese effects such as nausea and vomiting.

The present results supported the efficacy of carbamezapine in the treatment of trigeminal neuralgia as the pain was satisfactorily controlled by carbamazepine in $100 \%(n=9)$ of our patients. Pain perception after treatmentwas significantly decreased $(p=0.007)$ in comparison to pain perception before treatment with carbamazepine. However, there was no complete relief of pain with carbamazepine therapy in all of our patients. This was comparable to the results from a previous study (23). The present study had some limitations. Majority of clinical files of patients did not have any records of baseline data for comparison with the results from laboratory tests that were performed subsequently with the commencement of therapy. Factors that might affect the level of hepatic enzymes, such as weight gain, changes of metabolic parameters like glucose and serum lipids were not taken into consideration in the present study. Evaluation of pain outcome could be performed only on a limited number of our cases. This resulted in a limited opportunity to investigate the stratification of pain perception with adequate statistical power. Selection bias could not be completely excluded due to the retrospective nature of our study.

Carbamazepine should not be administered to patients with known hypersensitivity to carbamazepine, or to any of the tricyclic compounds, such as amitriptyline, trimipramine, imipramine, or 
their analogues or metabolites, in any forms whether in tablets or suspension because of their similarity in chemical structure. Besides that, baseline and periodic evaluations of haematological status, hepatic and renal function must be performed, especially in elderly patients and patients with history of liver disease. Patients manifesting a tendency towards anaemia and neutropenia may be supplemented with folic acid and lithium after consultation with the patient's primary care physician. Folic acid is a safe drug that may be administered safely in patients receiving carbamazepine as it reduces the risk of developing anaemia and leukopenia while lithium promotes WBC production $(16,17)$. Carbamazepine should be stopped immediately in cases of active liver disease or aggravated liver dysfunction. Potential correlations of the medical conditions and other medications taken by the patients and its associations with the drugs need to be explored.

\section{Conclusion}

The results of our study showed that there were no significant haematological, hepatic, renal, and nonspecific adverse effects associated with the use of carbamazepine. The efficacy of this drug in the treatment of trigeminal neuralgia was proven to be satisfactory. The benefits of using this drug certainly outweighed its adverse effects. It may be concluded that carbamazepine could be considered as the drug of choice in the treatment of trigeminal neuralgia.

\section{Declaration of interest}

The authors declare no conflicts of interest for this research

\section{Acknowledgement}

We would like to thank our supervisor, Associate Professor. Dr. Thomas George Kallarakkal for his valued guidance throughout the period of the study; Dr. Norlide and Mrs. Nabillah for her expert statistical advice and all patients who participated in this study.

\section{References:}

1. Punyani SR, Jasuja VR. Trigeminal neuralgia: An insight into the current treatment modalities. J Oral Biol Craniofac Res. 2012; 2(3): 188-97.

2. Toda K. Trigeminal Neuralgia-Symptoms, Diagnosis, Classification, and Related Disorders_Int J Oral. 2007; 4(1): 1-9.

3. Krafft RM. Trigeminal neuralgia. Am Fam Physician. 2008; 77(9): 1291-6.
4. Sindrup SH, Jensen TS. Pharmacotherapy of trigeminal neuralgia. Clin J Pain. 2002; 18(1): 22-7.

5. Chole R, Patil R, Degwekar SS, Bhowate RR. Drug treatment of trigeminal neuralgia: a systematic review of the literature. J Oral Maxillofac Surg. 2007; 65(1): 40-5.

6. Sobotka JL, Alexander B, Cook BL. A review of carbamazepine's hematologic reactions and monitoring recommendations. DICP. 1990; 24(12): 1214-9.

7. Younger J, McCue R, Mackey S. Pain outcomes: a brief review of instruments and techniques. Curr Pain Headache Rep. 2009; 13(1): 39-43.

8. Taylor LJ, Herr K. Pain intensity assessment: a comparison of selected pain intensity scales for use in cognitively intact and cognitively impaired African American older adults. Pain Manag Nurs. 2003; 4(2): 87-95.

9. Cruccu G, Sommer C, Anand P, Attal N, Baron R, Garcia-Larrea L, Haanpaa M, Jensen TS, Serra $J$, Treede RD. EFNS guidelines on neuropathic pain assessment: revised 2009. Eur J Neurol. 2010; 17(8): 1010-18.

10. Wong DL, Baker CM. Pain in children: comparison of assessment scales. Pediatr Nurs.1988; 14(1): 9-17.

11. Fink R. Pain assessment: the cornerstone to optimal pain management. Proceedings (Baylor University. Medical Center). 2000; 13(3): 236.

12. Scrivani SJ, Mathews ES, Maciewicz RJ. Trigeminal neuralgia. Oral Surg Oral Med Oral Pathol Oral Radiol Endod. 2005; 100(5): 52738.

13. Loh HS, Ling SY, Shanmuhasuntharam $P$, Zain $\mathrm{R}$, Yeo JF, Khoo SP. Trigeminal neuraligia. A retrospective survey of a sample of patients in Singapore and Malaysia. Aust Dent J. 1998; 43(3): 188-91.

14. Zakrzewska JM, Chaudhry Z, Nurmikko TJ, Patton DW, Mullens EL. Lamotrigine (lamictal) in refractory trigeminal neuralgia: results from a double-blind placebo controlled crossover trial. PAIN.1997; 73(2): 223-30.

15. Suwalsky M, Mennickent $S$, Norris B, Villena F, Sotomayor CP. Effects of the antiepileptic drug carbamazepine on human erythrocytes. Toxicol in vitro. 2006; 20(8): 1363-9.

16. Asadi-Pooya AA, Ghetmiri E. Folic acid supplementation reduces the development of some blood cell abnormalities in children receiving carbamazepine. Epilepsy \& Behavior. 2006; 8(1): 228-31. 
17. Oyesanmi O, Kunkel EJ, Monti DA, Field HL. Hematologic side effects of psychotropics. Psychosomatics.1999; 40(5): 414-21.

18. Tekce H, Kin Tekce B, Aktas G, Tanrisev M, Sit $M$. The evaluation of red cell distribution width in chronic hemodialysis patients. Int J Nephrol 2014. doi: $10.1155 / 2014 / 754370$

19. Clark VL, Kruse JA. Clinical methods: the history, physical, and laboratory examinations. JAMA. 1990; 264(21): 2808-9.

20. Haase MR. Carbamazepine-Induced Hepatorenal Failure in a Child. Pharmacotherapy.1999; 19(5): 667-71.

21. de Bragança AC, Moyses Z P, Magaldi AJ. Carbamazepine can induce kidney water absorption by increasing aquaporin 2 expression. Nephrol Dial Transplant. 2010; 25(12): 3840-5.
22. Jensen TS. Anticonvulsants in neuropathic pain: rationale and clinical evidence. Eur J Pain. 2002; 6(SA): 61-8.

23. Taylor JC, Brauer S, Espir ML. Long-term treatment of trigeminal neuralgia with carbamazepine. Postgraduate Medical J. 1981; 57(663): 16-8.

\section{Corresponding author:}

Assoc. Prof. Dr. Thomas George Kallarakkal

Department of Oral and Maxillofacial Clinical

Sciences,

Faculty of Dentistry,

University of Malaya,

50603 Kuala Lumpur,

Malaysia.

Email:thomasg22@um.edu.my

Tel: 019-3538434 